\title{
Paquimetria ultra-sônica da córnea em leporinos das raças Nova Zelândia (albino e vermelho) e Califórnia e eqüinos mestiços
}

\section{Corneal digital caliper rule readings of New Zeland (Albino and red) and California rabbits and mixed-bred horses}

\author{
Hermes Rodrigues Gomes, ${ }^{*}$ Nilo Sérgio Troncoso Chaves, ${ }^{\star \star}$ Duvaldo Eurides, ${ }^{\star \star \star}$ Clausmir Zanetti Jacomini, ${ }^{\star \star \star \star}$

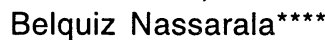

\begin{abstract}
Resumo
Foram estudados 30 olhos de leporinos, adultos, machos e fêmeas, das raças Nova Zelândia (albino e vermelho) e Califórnia cedidos pela EMATER/GO e abatidos artesanalmente e 30 olhos de eqüinos, adultos, machos e fêmeas, mestiços abatidos pelo Frigorífico Pomar S.A. Industrial e Comercial de Araguari, MG. Os olhos enucleados e os direitos foram acondicionados, individualmente, em câmaras úmidas (Filatov) com solução fisiológica 0,9\% e mantidos em caixa de isopor por um período máximo de seis horas. Cada olho foi dividido imaginariamente em cinco áreas (central, nasal superior, nasal inferior, temporal superior e temporal inferior). As leituras digitais com o paquímetro ultra-sônico de Storz foram feitas em três pontos para cada área, totalizando 15 vezes em cada olho. A média geral obtida para os leporinos foi de $435,35 \mu \pm 27,59 \mu$. Os valores médios foram diferentes para cada ponto, sendo que a média obtida na área nasal inferior $(423,67 \mu \pm 23,93 \mu)$ foi a menos espessa, e na temporal superior $(447,37 \mu \pm 56,46 \mu)$, a mais espessa; para os eqüinos, a média geral obtida foi de $896,79 \mu \pm 30,15 \mu$, a média obtida na área temporal inferior $(884,87 \mu \pm 49,13 \mu)$ foi a menos espessa e a nasal inferior $(916,07 \mu \pm 34,14 \mu)$ a mais espessa. O método foi eficiente, seguro e simples. Os valores obtidos são inéditos, mas só servem como valores de referência para estas amostras, pois são dependentes da idade, do peso, do sexo, da raça, da dieta e até do horário do exame, variáveis estas que não foram avaliadas neste trabalho.
\end{abstract}

Palavras-chave: paquimetria ultra-sônica, paquímetro de Storz, leporinos, eqüinos, olhos.

\begin{abstract}
Thirty eyes from adult rabbit males and females of New Zealand and California breeds (albino and red) were studied, as well as thirty eyes of adult male and female horses of undefined breed slughtered at the Frigorífico Pomar S.A. Industrial e Comercial of Araguari, of Minas Gerais State, Brazil. The enucleated eyes and the rigths were maiteinede separately in fresh recipient (Filatov) in $0,9 \%$ saline solution and kep within na isothermal box for a period no longer than 6 hours. Five different areas were it was considered as follows: central, upper nasal, lower nasal, upper temporal and lower temporal. The digital readings with the Storz's ulstra sonic caliper rule were performed in three points for each area, in a total of 15 readings for each eye. The general average results obtained for rabbits were $435.35 \mu \pm 27.59 \mu$, but everage values were different for each site; sthe lower nasal area $(423.67 \mu \pm 56.4 \mu)$ was the thinnest area and the upper temporal $(447.37 \mu \pm 56.4 \mu)$ was the tickest. For horses, the average was $896.79 \mu \pm 30.15 \mu$, although average values were also different for each site. The lower temporal ares $(884.87 \mu \pm$ $49.13 \mu)$ was the tinnest and the lower nasal area $(916.07 \mu \pm 34.14 \mu)$ the thickest one. The method was efficient, safe and simple. Although this is the report od such measurements, but they are considered to be valid only for these samples, once it depends on other factors such as age, weight, beed, diet and time of examination, which were not evaluated in this study.
\end{abstract}

Keywords: Storz's ultra-sonic caliper rule, rabbit, equine, eye.

\section{Introdução}

O exame oftálmico vem-se constituindo num excelente método para o auxílio no diagnóstico de doenças em animais e no homem, sobretudo as zoonoses. Portanto, é necessária a compreeensão da anatomia e dos fenômenos fisiológicos dos olhos das diversas espécies domésticas, estabelecidos através de sistematização científica e dos seus valores de referência. Além disto, sendo a córnea um sítio privilegiado para transplantes, torna-se necessário o conhecimento detaIhado de toda a sua estrutura, para o êxito dos transplantes xenógenos. Poucos trabalhos foram feitos com a paquimetria

\footnotetext{
* Professor Assistente - Depto. de Medicina Veterinária-EV/UFG - Escola de Veterinária - Caixa Postal 131. - 74001-970 - Goiânia, GO

* Professor titular - Depto de Medicina Veterinária -EV/UFG

*** Professor Titular - Curso de Medicina Veterinária - UFU

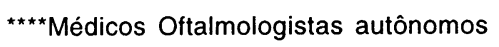


ultra-sônica na Medicina Veterinária. Em algumas espécies não há, sequer, os valores de referência.

A paquimetria ultra-sônica é utilizada na rotina do exame oftálmico para a determinação de valores fisiológicos, bem como alterações da espessura corneana, tais como: distrofias endoteliais, trauma por cirurgia intra-oculares e uso de lentes de contato. Na Medicina, a paquimetria da córnea é essencial no planejamento das cirurgias refrativas e o aumento da sua espessura pode estar relacionado com o seu grau de hidratação que revela a estimativa da reserva funcional do seu endotélio.

Existem quatro métodos para se realizar a paquimetria de aplanação: óptico; interferometria; eletromecânico e ultra-sônico.

Fundamentos da paquimetria ultra-sônica - $O$ princípio da paquimetria ultra-sônica é ultra-sonográfico (modo A) e o instrumento mede o tempo necessário para que o som (freqüência de 10 a $20 \mathrm{Mhz}$, comprimento de onda $150 \mathrm{~mm}$ ) passe do final do transdutor à membrana de Descemet e retorne ao transdutor. A espessura da córnea é dada em micra pela seguinte fórmula $-(C=t \times V / 2)$, onde: $C=$ espessura corneana, $\mathrm{t}=$ tempo de trânsito do som e $\mathrm{V}=$ Velocidade de propagação do som na córnea (Belfort e Kara-José, 1997).

O paquímetro óptico apresenta as seguintes desvantagens: dificuldade no alinhamento preciso ao longo da curvatura corneana, necessidade de uma tabela de correção dos valores e treinamento e cuidado do técnico para a obtenção de medidas precisas. O método por interferometria com laser não está disponível clinicamente. 0 método eletromecânico é menos prático e de exame mais demorado, além de não poder ser realizado com o paciente deitado. Já a paquimetria ultra-sônica é a preferida devido a facilidade de utilização, precisão, por ser o aparelho portátil e não há diferença de medição da espessura entre 0 olho direito e o esquerdo. Por medir a espessura corneana excentricamante, sem o uso da lâmpada de fenda, o paciente pode estar sentado ou em decúbito dorsal (Belfort e Kara-José,1997).

Belfort e Kara-José (1997) descreveram que a espessura da córnea foi medida, pela primeira vez, in vivo, em 1880 e foram usados na oportunidade dois microscópicos de igual poder, alinhados horizontalmente em um ângulo de 40 graus.

Gilger et al. (1991) mediram as espessuras de córneas normais de 150 olhos de cães com câmaras anteriores e PIO normais, usando valores obtidos da parte central, periférica, temporal e superior deste órgão. O valor médio obtido foi de $562 \pm 6,2 \mu$ As áreas periféricas foram mais espessas do que a área central e esta diferença aumentou com a idade e com o peso do animal. Fêmeas apresentaram córneas significativamente mais delgadas.

Gilger et al. (1993) estudaram a espessura periférica, temporal e central de 35 gatos com córneas normais, câmaras anteriores e pressões intra-oculares normais. $O$ valor médio central foi de $578 \pm 64 \mathrm{~m}$ e não existiram diferenças significativas de acordo com as posições e nem com respeito ao sexo, porém, as espessuras variaram significativamente com a idade, quando foram aferidas as córneas dos animais com até 100 meses de idade.

Kudo et al. (1996) pesquisaram a espessura da córnea de 53 cães adultos, de pesos variados, com córneas, câmaras anteriores e PIO normais. Observaram que a espessura do centro da córnea foi mais delgada do que a espessura periférica inferior, temporal e nasal.
Schoster, Wickman, Stuhr (1995) avaliaram a espessura de córneas de 25 gatos em 13 pontos diferentes, através de paquimetria ultra-sônica e encontraram um perfil heterogêneo. Os valores temporais mostraram-se mais espessos, os valores nasais foram os menos espessos e não houve relação dos valores obtidos com o peso e sexo.

\section{Material e métodos}

Foram utilizados 30 olhos de leporinos das raças Nova Zelândia (albino e vermelho) e Califórnia, machos e fêmeas, adultos, mestiços, abatidos artesanalmente e 30 olhos de eqüinos, adultos, machos e fêmeas, mestiços abatidos pelo Frigorífico Pomar S.A. Industrial e Comercial de Araguari, MG. Os olhos foram imediatamente enucleados e mantidos em câmara úmida, com solução de $\mathrm{NaCl} 0,9 \%$, por um período de no máximo seis horas, acondicionados em câmara de Filatov (Figura 1) e transportados em caixa de isopor fechada até o Instituto de Olhos de Goiânia onde foram feitas as paquimetrias. Em cada olho direito tomou-se três medidas digitais, com paquímetro ultra-sônico Storz' ${ }^{1}$ para cada ponto: central, nasal superior, nasal inferior temporal superior, temporal inferior, totalizando 15 medidas (Figura 2 e Figura 3 ).

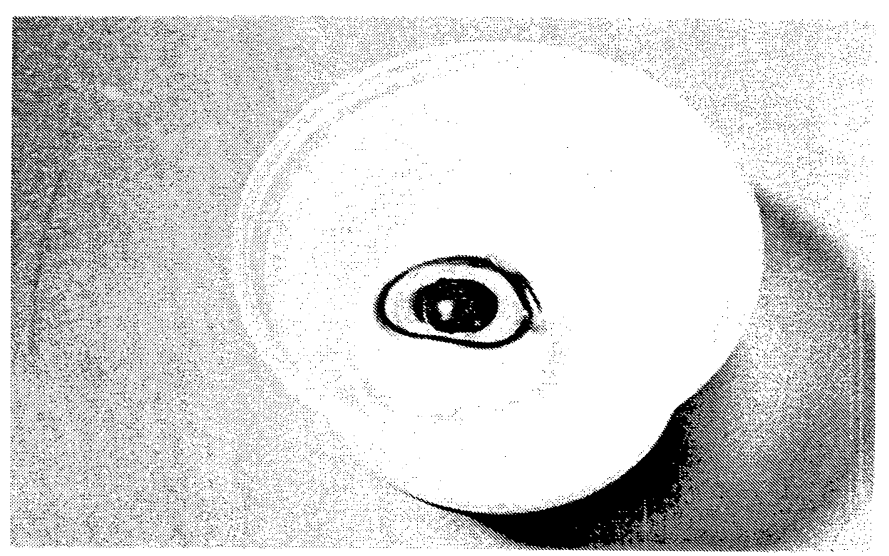

Figura 1 - Globo ocular mantido em câmara úmida

NS

TS

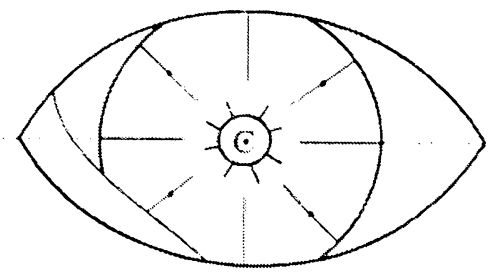

NI

TI

Figura 2 - Esquema de divisão do olho nas áreas nasal superior (NS), temporal superior (TS), nasal inferior (NI) temporal inferior (TI) e central (C)

\footnotetext{
1 Paquímetro ultra-sônico Storz - USA.
} 


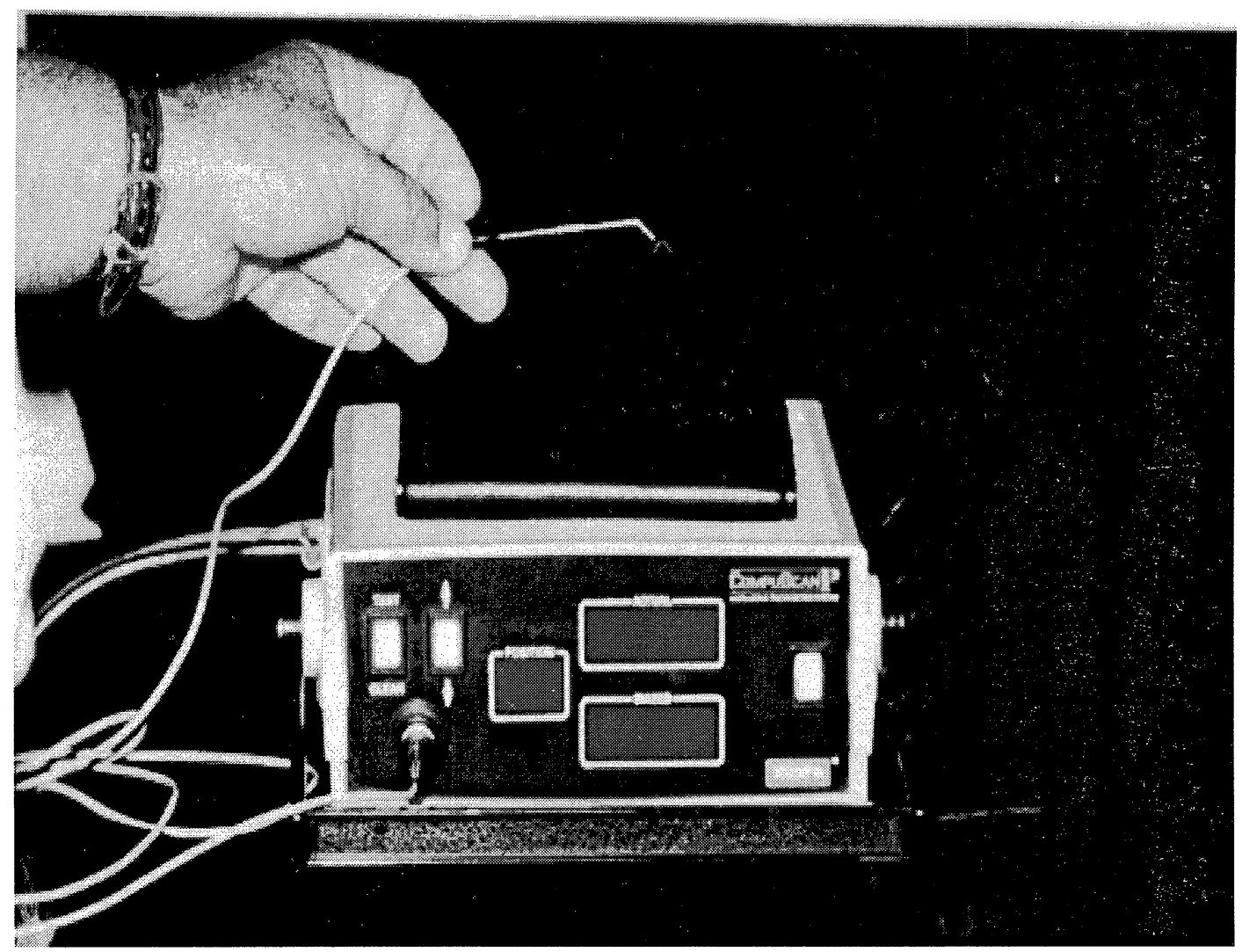

Figura 3 - Paquímetro ultra-sônico de Storz

\section{Resultados}

As Tabelas 1 e 2 demonstram os resultados individuais, em micra, após a aferição feitas com o paquímetro ultra-sônico de Storz, nas áreas central, temporal superior e inferior e nasal superior e inferior nos olhos de 30 leporinos e. 30 equinos, além da média por animal e por pontos.

\section{Discussão e conclusões}

Os valores centrais e periféricos obtidos através da paquimetria ultra-sônica com o paquímetro de Storz, nas córneas dos leporinos e eqüinos, neste trabalho, foram muito heterogêneos, ora coincidem, ora não, com os valores encontrados por Gilger et al. (1991) em cães, Gilger et al. (1993) em gatos, Schoster, Wickman, Stuhr (1995), em gatos, Kudo et al. (1996) em cães. Os pontos mais espessos e menos espessos encontrados nas córneas dos leporinos e dos eqüinos foram, respectivamente, $444,37 \mu \pm 48,00 \mu$ (temporal inferior) e $423,67 \mu \pm 23,93 \mu$ (nasal inferior); $916,07 \mu \pm$ $43,14 \mu$ (nasal inferior) e $884,97 \mu \pm 49,13 \mu$ (temporal inferior). As variações encontradas pelos mesmos autores em relação a idade, sexo e
Tabela 1 - Valores médios, em micra, de cinco pontos e da média geral, obtidos por meio da paquimetria ultra-sônica nos olhos de leporinos raça Nova Zelândia. Goiânia, julho, 1998

\begin{tabular}{|c|c|c|c|c|c|c|}
\hline & & \multicolumn{2}{|c|}{ TEMPORAL } & \multicolumn{2}{|c|}{ NASAL } & \multirow[b]{2}{*}{ MÉDIA } \\
\hline $\mathrm{OLHO}$ & CENTRAL & SUPERIOR & INFERIOR & INFERIOR & SUPERIOR & \\
\hline 1 & 404 & 394 & 402 & 440 & 391 & 406,2 \\
\hline 2 & 412 & 413 & 494 & 490 & 404 & 442,6 \\
\hline 3 & 408 & 411 & 395 & 406 & 415 & 407 \\
\hline 4 & 411 & 409 & 410 & 407 & 402 & 407,8 \\
\hline 5 & 469 & 437 & 476 & 430 & 444 & 451,2 \\
\hline 6 & 497 & 615 & 614 & 459 & 482 & 533,4 \\
\hline 7 & 402 & 394 & 412 & 394 & 413 & 403 \\
\hline 8 & 411 & 412 & 391 & 419 & 420 & 410,6 \\
\hline 9 & 464 & 646 & 454 & 411 & 449 & 484,8 \\
\hline 10 & 467 & 441 & 460 & 422 & 444 & 446,8 \\
\hline 11 & 441 & 396 & 410 & 440 & 410 & 419,4 \\
\hline 12 & 426 & 413 & 418 & 411 & 417 & 417 \\
\hline 13 & 449 & 454 & 456 & 428 & 441 & 445,6 \\
\hline 14 & 427 & 428 & 413 & 441 & 410 & 423,8 \\
\hline 15 & 444 & 449 & 440 & 423 & 428 & 436,8 \\
\hline 16 & 431 & 416 & 434 & 416 & 412 & 421,8 \\
\hline 17 & 432 & 432 & 428 & 396 & 428 & 423,2 \\
\hline 18 & 428 & 429 & 455 & 392 & 434 & 427,6 \\
\hline 19 & 439 & 452 & 477 & 391 & 424 & 436,6 \\
\hline 20 & 432 & 425 & 418 & 399 & 431 & 421 \\
\hline 21 & 420 & 411 & 419 & 413 & 416 & 415,8 \\
\hline 22 & 429 & 411 & 419 & 413 & 416 & 417,6 \\
\hline 23 & 426 & 499 & 471 & 413 & 443 & 450,4 \\
\hline 24 & 419 & 420 & 408 & 393 & 405 & 409 \\
\hline 25 & 444 & 442 & 434 & 427 & 443 & 438 \\
\hline 26 & 439 & 428 & 436 & 439 & 427 & 433,8 \\
\hline 27 & 465 & 473 & 476 & 469 & 448 & 466,2 \\
\hline 28 & 452 & 469 & 565 & 440 & 427 & 470,6 \\
\hline 29 & 440 & 453 & 465 & 438 & 424 & 444 \\
\hline 30 & 444 & 459 & 465 & 450 & 426 & 448,8 \\
\hline Média & 435,73 & 444,37 & 447,17 & 423,67 & 425,80 & 435,35 \\
\hline Desvio-padrão & 21,86 & 56,46 & 48,00 & 23,93 & 18,33 & 27,59 \\
\hline Erro padrão & 3,99 & 10,31 & 8,76 & 4,37 & 3,35 & 5,04 \\
\hline Int. de conf."t" & 8,18 & 21,13 & 17,97 & 8,96 & 6,86 & 10,33 \\
\hline Limite inferior & 427,55 & 423,24 & 429,20 & 414,71 & 418,94 & 425,02 \\
\hline Limite superior & 443,91 & 465,50 & 465,13 & 432,62 & 432,66 & 445,67 \\
\hline
\end{tabular}


Tabela 2 - Valores médios, em micra, de cinco pontos e da média geral, obtidos por meio da paquimetria ultra-sônica nos olhos de eqüino mestiços. Goiânia, julho, 1998

\begin{tabular}{|c|c|c|c|c|c|c|}
\hline & & \multicolumn{2}{|c|}{ TEMPORAL } & \multicolumn{2}{|c|}{ NASAL } & \multirow[b]{2}{*}{ MÉDIA } \\
\hline $\mathrm{OLHO}$ & CENTRAL & SUPERIOR & INFERIOR & INFERIOR & SUPERIOR & \\
\hline 1 & 869 & 903 & 908 & 849 & 834 & 872,6 \\
\hline 2 & 864 & 899 & 889 & 942 & 906 & 900 \\
\hline 3 & 885 & 879 & 820 & 948 & 864 & 879,2 \\
\hline 4 & 917 & 891 & 864 & 926 & 893 & 898,2 \\
\hline 5 & 873 & 891 & 952 & 891 & 846 & 890,6 \\
\hline 6 & 842 & 869 & 942 & 935 & 816 & 880,8 \\
\hline 7 & 962 & 876 & 853 & 939 & 816 & 889,2 \\
\hline 8 & 911 & 925 & 943 & 953 & 948 & 936 \\
\hline 9 & 893 & 955 & 792 & 910 & 869 & 883,8 \\
\hline 10 & 946 & 967 & 940 & 959 & 963 & 955 \\
\hline 11 & 871 & 959 & 916 & 915 & 871 & 906,4 \\
\hline 12 & 880 & 899 & 843 & 934 & 856 & 882,4 \\
\hline 13 & 810 & 820 & 794 & 856 & 833 & 822,6 \\
\hline 14 & 837 & 842 & 879 & 901 & 834 & 858,6 \\
\hline 15 & 803 & 902 & 834 & 879 & 823 & 848,2 \\
\hline 16 & 916 & 929 & 909 & 957 & 941 & 930,4 \\
\hline 17 & 961 & 905 & 876 & 867 & 917 & 905,2 \\
\hline 18 & 815 & 868 & 919 & 855 & 864 & 864,2 \\
\hline 19 & 877 & 913 & 924 & 955 & 875 & 908,8 \\
\hline 20 & 945 & 958 & 830 & 931 & 910 & 914,8 \\
\hline 21 & 931 & 930 & 894 & 925 & 942 & 924,4 \\
\hline 22 & 895 & 902 & 954 & 935 & 890 & 915,2 \\
\hline 23 & 937 & 960 & 943 & 949 & 948 & 947,4 \\
\hline 24 & 823 & 831 & 884 & 871 & 906 & 863 \\
\hline 25 & 911 & 910 & 955 & 938 & 905 & 923,8 \\
\hline 26 & 898 & 937 & 891 & 934 & 907 & 913,4 \\
\hline 27 & 882 & 933 & 862 & 891 & 928 & 899,2 \\
\hline 28 & 872 & 872 & 803 & 883 & 885 & 863 \\
\hline 29 & 895 & 910 & 866 & 953 & 905 & 905,8 \\
\hline 30 & 947 & 952 & 870 & 901 & 938 & 921,6 \\
\hline Média & 888,93 & 906,23 & 884,97 & 916,07 & 887,77 & 896,79 \\
\hline Desvio-padrão & 44,55 & 38,60 & 49,13 & 34,14 & 43,07 & 30,15 \\
\hline Erro padrão & 8,13 & 7,05 & 8,97 & 6,23 & 7,86 & 5,50 \\
\hline Int. de conf." $t "$ & 16,67 & 14,45 & 18,39 & 12,78 & 16,12 & 11,28 \\
\hline Limite inferior & 872,26 & 891,79 & 866,58 & 903,29 & 871,64 & 885,51 \\
\hline Limite superior & 905,61 & 920,68 & 903,35 & 928,84 & 903,89 & 908,08 \\
\hline
\end{tabular}

peso não foram analisadas no presente trabalho, pois não havia padronização para estas variáveis.

Os valores individuais e médios obtidos neste trabalho, através da paquimetria Ultra-sônica, em leporinos $(435,35 \mu \pm 27,59 \mu)$ e em eqüinos $(896,79 \mu \pm 30,15 \mu)$, não foram confrontados com dados da mesma espécie, pois não encontramos literaturas disponíveis, nas quais pesquisadores tenham realizado trabalhos semelhantes. Portanto, os valores obtidos pela paquimetria ultra-sônica com o paquímetro de Storz, neste ensaio podem ser considerados como valores de referência apenas para as unidades experimentais nele contidas e são dados inéditos.

As espessuras se mostraram diferentes nos diversos pontos das córneas nos leporinos e nos eqüinos neste trabalho, bem como são diferentes aquelas da literatura referente a outras espécies, demostrando limitação física para intervenção cirúrgica, cuja intenção seja o transplante perfurante xenógeno.

O paquímetro ultra-sônico Storz foi de fácil manuseio, preciso e não se pôde avaliar se 0 aparelho provocou danos às córneas que pudessem influenciar nos resultados, pois as aferições foram feitas em olhos enucleados.

\section{Material da pesquisa}

Paquímetro Ultra-sônico de Storz - USA.

\section{Referências}

BELFORT, R., JOSÉ, N.K.. Cornea Clínica Cirúrgica. São Paulo: Roca, 1997. $607 \mathrm{p}$.

WITLEY, R.D., MCLAUGHIN, S.A., WRIGHT, J.C., Canine comeal thickness measured by ultrasonic pachymetry. American Journal of Veterinary Research. v. 52 n. 10, 1991, p.1570-1572.

GILGER, B.C., WRIGHT, J.C., WHITLEY, R.R., MCLAUGHLIN, Sa. Corned thickness measures by ultrasonic pachymetry in cats.
American Journal of Veterinary Research. v. 54, n. 2, 1993. p. 228-230.

KUDO, S., KANEMAKI, N., UCHIUM, N., NATSURA, K. The thickness of the cornea using an ultrasonic pachymeter in acult dogs. Journal Veterinary Medical Association. v. 49, n. 1, 1996, p. 32-34.

SCHOSTER, J.V., WICKMAN, L., STUHR, C. The use ultrasonic pachymetry and computer enhancement to illustrate the collective corneal thickness profile of 25 cats. Veterinary and comparative opthalmology, v. 5, n. 2. 